\title{
Clasificación Pedológica en la Subcuenca del Río Zaratí para el Desarrollo de Buenas Prácticas de Conservación de los Suelos y Aguas
}

\author{
González, Digna \\ Universidad Tecnológica de Panamá, Panamá \\ digna.gonzalez@utp.ac.pa \\ Santana, Carlos \\ Universidad Tecnológica de Panamá, Panamá \\ carlos.santana@utp.ac.pa \\ Fábrega, José \\ Centro de Invest. Hidráulicas e Hidrotécnicas (CIHH) - UTP Panamá, Panamá \\ jose.fabrega@utp.ac.pa \\ Leiva, Jorge \\ Universidad de Costa Rica , San José, Costa Rica \\ jorge.leivasanabria@ucr.ac.cr \\ Muñoz, Melisabel \\ Grupo de investigación Water Management and Hydrological Sciences - UTP Panamá, Panamá \\ melisabel.munoz@utp.ac.pa
}

\begin{abstract}
Pedology studies the genesis and taxonomic classification of soils to evaluate and make adequate recommendations for its improvement and conservation. This research conducted a pedological study in the Zarati River Sub-basin (SRZ) to analyze the evolution, the development of soil profiles and characterize their morphological, physical, chemical, and biological properties. The technique used was based on data collection through borehole observations at a depth of $120 \mathrm{~cm}$, soil sampling in observation pits and subsequent laboratory analysis. In total, 14 modal profiles or representative soils were identified and classified according to the Soil Taxonomy system of the United States Department of Agriculture. The soils in the SRZ are ancient soils, which show natural and anthropic degradation that affects environmental quality, aquifer recharge and its potential yield for agricultural activities. In the SRZ there are three dominant soil orders: Ultisols, Inceptisols, and Entisols,
\end{abstract}


distributed in a landscape catena from the lower, middle and upper altitudinal ranges of the SRZ, respectively. According to the classification of land use capacity of the dominant soils, they are mostly classified as class $\mathrm{V}$ and class $\mathrm{VI}$ lands. This research recommends to implement intensive conservation practices for soils and water managements such as deep mechanization to enhance infiltration rates, tree planting and perennial crops to protect the soil from the direct impact of rain to control erosion and soil deterioration.

Keywords: Pedology, taxonomy, inceptisol, ultisol, entisol.

\section{RESUMEN}

La pedología se encarga de estudiar la clasificación taxonómica permitiendo conocer el estado actual del suelo para evaluar y realizar recomendaciones adecuadas para su mejoramiento y su conservación. Esta investigación llevo a cabo un estudio pedológico en la Subcuenca del Río Zaratí (SRZ) para conocer la evolución, el desarrollo de los diferentes perfiles y analizar sus propiedades morfológicas, físicas, químicas y biológicas. La metodología utilizada se basó en la recolección de datos mediante observaciones de barreno a $120 \mathrm{~cm}$ de profundidad, muestreo de suelo en fosas de observación, pruebas de laboratorio y el posterior análisis de resultados. Se identificaron 14 perfiles modales o suelos representativos y se clasificaron según el sistema de Taxonomía de Suelos del Departamento de Agricultura de los Estados Unidos y se desarrolló la clasificación según la capacidad de uso de suelo. Los suelos en la SRZ son suelos antiguos, que presentan degradación natural y antrópica que afecta la calidad ambiental, la recarga acuífera y su rendimiento para actividades agrícolas. En la SRZ existen tres órdenes de suelos dominantes: Ultisoles, e Inceptisoles asociados a Entisoles, distribuidos en la parte baja, media y alta de la SRZ, respectivamente. Según la capacidad de uso de suelo se clasifican en tierras clase V y VI. Se recomienda implementar prácticas intensivas de manejo y conservación de suelos y agua como mecanización profunda, siembra de árboles y cultivos perennes que protejan el suelo del impacto directo de la lluvia para el control de la erosión y deterioro del suelo.

Palabras claves: Pedología, taxonomía, inceptisol, ultisol, entisol.

\section{INTRODUCCIÓN}

En Panamá, la información científica sobre las propiedades y las condiciones en las que se encuentra el suelo es insuficiente. Entender el estado actual de los suelos promueve 
indirectamente la conservación del recurso hídrico.

La clasificación taxonómica de los suelos nos conduce al estudio de la formación y evolución de este, además, el desarrollo de los diferentes perfiles y tipos de suelo. Por tanto, la clasificación de suelos se basa en diferentes criterios que involucran las características intrínsecas del suelo dependientes de los procesos pedogenéticos, las propiedades físicas y químicas que relacionan los factores de formación y aptitud de uso de suelo.

El propósito de la clasificación es organizar los tipos de suelo para que a futuro la población tenga claro los usos que pueda darse a un territorio, para poder desarrollar una planificación óptima de acuerdo con sus características y así llevar a cabo actividades productivas sustentables, proteger los recursos hídricos y conservar a su vez la biodiversidad.

Esta investigación se desarrolla en la subcuenca del río Zaratí (SRZ), comprendida por un área de 168.8 km2 y una población de aproximadamente 22,475 habitantes. En la parte baja de la subcuenca se localiza la ciudad de Penonomé, cabecera de la provincia de Coclé. En la SRZ y en sus alrededores se desarrollan actividades agrícolas y ganaderas, lo cual aunado a la demanda de agua para consumo humano, tiene un impacto significativo en el recurso hídrico de la región. Con miras a la conservación de los suelos y del recurso hídrico del área de estudio, la presente investigación pretende clasificar los suelos según la Soil Taxonomy usando la metodología del Departamento de Agricultura de los Estados Unidos (USDA) [1]. Este trabajo será una herramienta que aporte a la consulta del estudio de suelos, brindando información cartográfica y taxonómica de los suelos para lograr un conocimiento integrado del territorio.

\section{MÉTODO}

\section{A. Selección sitios de muestreo.}

Se delimitó la subcuenca y se subdividió en 3 secciones representativas para lograr una mejor estimación (parte baja, media y alta, según sus rangos altitudinales). Inicialmente, se establecieron transectos definidos realizando aproximadamente una observación por cada $\mathrm{km} 2$, donde las observaciones se realizaron con un barreno de tipo holandés, el cual es una herramienta helicoidal rotatoria de 1.20 metros de profundidad que permitió exponer los horizontes del perfil del suelo en la superficie para ser descritos y analizados.

Con la información recopilada se logró conocer las áreas representativas y similares en estratigrafía según la moda y se estableció la ubicación de los puntos de muestreo de los suelos dominantes (calicata).

\section{B. Apertura de calicatas, descripción in situ y recolección de muestras.}

Se realizó la excavación de la calicata con pica y pala. Una calicata es una fosa de observación utilizada para exponer el perfil del suelo de manera vertical. 
Seguidamente se procedió con la descripción del paisaje circundante del sitio donde está ubicado el perfil de observación, separación y definición de los horizontes maestros para su posterior descripción. Para la descripción de los horizontes se tomó en cuenta la textura, color en húmedo, color en seco, estructura y consistencia, pruebas especiales ( $\mathrm{NaF}$, $\mathrm{Mn}, \mathrm{HCl})$, poros y raíces.

Se tomó una muestra de suelo en cada horizonte, la muestra se recolectó en anillos de acero de $1 \mathrm{~cm}$ de alto por $5.5 \mathrm{~cm}$ de diámetro (dos muestras de suelo) y otro de $5 \mathrm{~cm}$ de alto por $5.5 \mathrm{~cm}$ de diámetro (una muestra de suelo) lo que evita que la muestras sea disturbada.

\section{Pruebas físicas y químicas.}
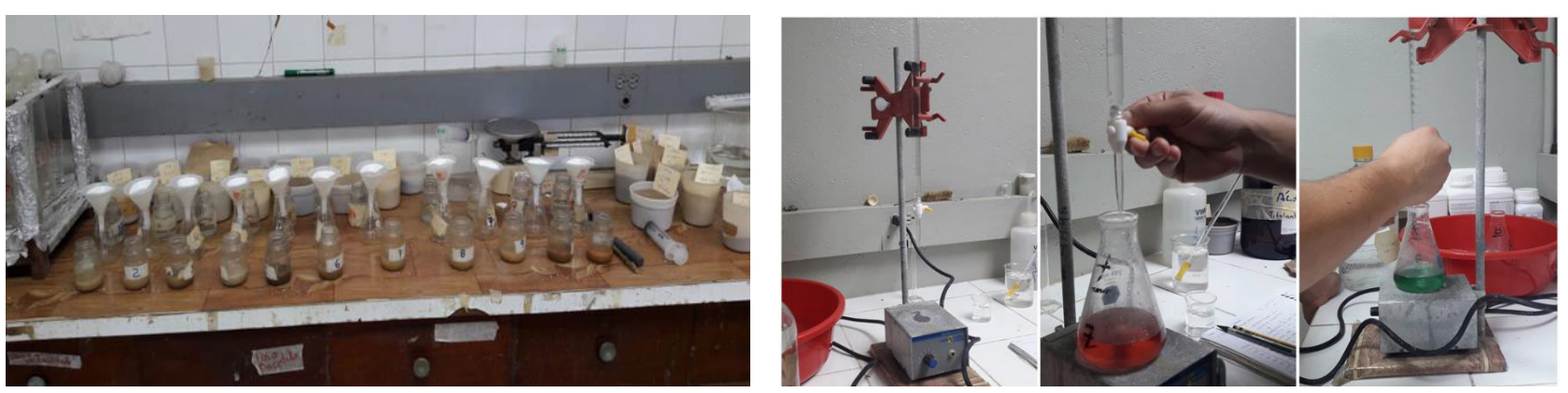

Fig. 1. Determinación de capacidad de intercambio catiónico.

Se realizó un análisis de acuerdo con las características físicas y químicas del suelo obtenidas mediante las pruebas realizadas en el laboratorio de suelos del Instituto de Investigaciones Agropecuarias (IDIAP). Las pruebas físicas fueron:textura, densidad aparente, densidad real y conductividad hidráulica y las pruebas químicas: bases intercambiables y capacidad de intercambio catiónico, potencial de hidrógeno $(\mathrm{pH})$, porcentaje de materia orgánica (\%MO), Fósforo (P), Calcio (Ca), Magnesio (Mg), Aluminio (Al), Potasio (K), Hierro $(\mathrm{Fe})$, Manganeso (Mn), Zinc (Zn). Cada una de estas características son utilizadas para determinar la clasificación taxonómica del suelo, la cual busca identificar los suelos con una génesis similar y utilizar un criterio específico para agruparlos.

\section{Clasificación Taxonómica}

Una vez se conocieron las características de cada perfil se facilitó la clasificación taxonómica del suelo jerarquizada, de modo que cada suelo se fue analizando y comparando, lo que permitió agruparlo en una categoría general (órdenes), la cual, alberga más categorías (Subórdenes). Se realizó utilizando la metodología del Departamento de Agricultura de los Estados Unidos (USDA) (Soil Survey Staff, 1999, 2014) [1, 2] que clasifica el suelo utilizando los doce ordenes de suelo que son: Entisoles, Inceptisoles, Histosoles, Aridisoles, Vertisoles, 
Espodosoles, Andisoles, Mollisoles, Gelisoles, Alfisoles, Ultisoles y Oxisoles.

\section{E. Clasificación según la Capacidad de uso de suelo.}

La metodología que se utilizó se fundamentó en un sistema estructurado en clase, subclase y unidades de manejo que permite analizar las condiciones del suelo, con el fin de evitar y/o disminuir su degradación [3].

Según su clase se dividen en:

- I, II, III, IV (grupo de tierras con capacidad para ser utilizadas en agricultura y ganadería tecnificada).

- V, VI, VII (grupo de tierras que pueden ser utilizadas en forma restringidas para cultivos semipermanentes o permanentes).

- VIII (utilizadas sólo para preservación, conservación y ecoturismo).

Según subclases de acuerdo con factores limitantes como: erosión (e), suelo (s), drenaje (d), clima (c).

\section{RESULTADOS}

Tabla 1. ÓRDENES DEL SUELO [2].

\begin{tabular}{|c|c|c|}
\hline Ubicación & Orden & Descripción característica \\
\hline $\begin{array}{l}\text { Las Lomas Abajo, Cerro Gordo, San- } \\
\text { ta Cruz, El Potrero, Los Llanitos, Monte } \\
\text { Grande, Pajonal Centro, El Guabal. }\end{array}$ & Ultisoles & $\begin{array}{l}\text { Suelos que presentan acumulación iluvial de arci- } \\
\text { lla,óxidos de Fe y Al en el horizonte subsuperficial } \\
\text { B (argílico). Son suelos plenamente desarrollados, } \\
\text { antiguos, ya que los materiales parentales han } \\
\text { sido } \\
\text { meteorizados por mucho tiempo. }\end{array}$ \\
\hline Paso Ancho, Membrillo Arriba, Sofre. & Inceptisoles & $\begin{array}{l}\text { Son suelos de desarrollo incipiente, con presencia } \\
\text { de un horizonte B de tipo cámbico, con desarrollo } \\
\text { de estructura y color rojizo. Se localizan por lo ge- } \\
\text { neral en } \\
\text { posiciones topográficas extremas. }\end{array}$ \\
\hline Cerro Turega, Las Pailas, El Coquillo. & Entisoles & $\begin{array}{l}\text { Suelos relativamente recientes, con poco desarro- } \\
\text { llo de horizontes o capas, comunes en paisajes de } \\
\text { inundación, topografía escarpada y sitios de } \\
\text { deposición de material por erosión hídrica. }\end{array}$ \\
\hline
\end{tabular}


Tabla 2. Clasificación taxonómica USDA y datos físicos y químicos seleccionados [1].

\begin{tabular}{|c|c|c|c|c|c|c|c|c|}
\hline Sitio & Orden & Suborden & Gran Grupo & $\begin{array}{l}\text { Altitud } \\
\text { (msnm) }\end{array}$ & $\begin{array}{l}\text { Prof. hte. A } \\
(\mathrm{cm})\end{array}$ & $\begin{array}{c}\text { CIC } \\
\text { (hte.B) }\end{array}$ & $\begin{array}{c}\% \mathrm{SB} \\
\text { (hte.B) }\end{array}$ & $\begin{array}{c}\mathrm{K}_{\text {sat }} \\
\text { (hte.B) }\end{array}$ \\
\hline \multicolumn{9}{|c|}{ Parte Baja } \\
\hline Paso Ancho (ZT-06) & Inceptisol & Ustepts & Dystrustetps & 40 & 11 & 21,6 & 53 & 131,64 \\
\hline Las Lomas Abajo (ZT-22) & Ultisol & Aquults & Plinthaquults & 57 & 20 & 18,4 & 38 & 0,00 \\
\hline Cerro Gordo (ZT-30) & Ultisol & Aquults & Epiaquults & 49 & 5 & 32,4 & 18 & 1,46 \\
\hline \multicolumn{9}{|c|}{ Parte Media } \\
\hline Santa Cruz (ZT-46) & Ultisol & Ustults & Plinthustults & 328 & 22 & 33,6 & 12 & 0,00 \\
\hline El Potrero (ZT-58) & Ultisol & Humults & Palehumults & 96 & 12 & 15,2 & 31 & 64,15 \\
\hline Los Llanitos (ZT-71) & Ultisol & Ustults & Haplustults & 179 & 23 & 18,8 & 17 & 0,00 \\
\hline Monte Grande (ZT-128) & Ultisol & Ustults & Plinthustults & 149 & 8 & 30,0 & 18 & 0,00 \\
\hline \multicolumn{9}{|c|}{ Parte Alta } \\
\hline Pajonal Centro (ZT-115) & Ultisol & Aquults & Plinthaquults & 252 & 31 & 18 & 49 & 0,00 \\
\hline El Guabal (ZT-134) & Ultisol & Humults & Plinthohumults & 244 & 12 & 14,4 & 14 & 0,02 \\
\hline Membrillo Arriba (ZT-104) & Inceptisol & Ustepts & Humustepts & 511 & 23 & 19,2 & 7 & 0,01 \\
\hline Sofre (ZT-97) & Inceptisol & Ustepts & Humustepts & 332 & 24 & 19,2 & 22 & 0,25 \\
\hline Cerro Turega (ZT-133) & Entisol & Orthents & Udorthents & 407 & 28 & - & - & - \\
\hline Las Pailas (ZT-92) & Entisol & Orthents & Udorthents & 440 & 15 & - & - & - \\
\hline El Coquillo (ZT-99) & Entisol & Orthents & Udorthents & 685 & 7 & - & - & - \\
\hline
\end{tabular}

CIC: Capacidad de intercambio catiónico (cmol+/L). Ksat: Conductividad hidráulica (cm/h).

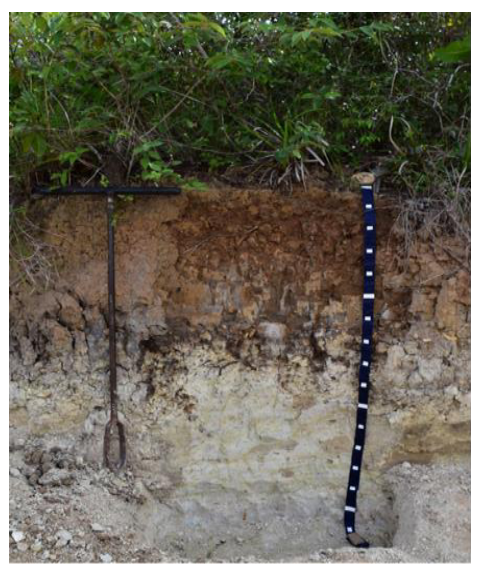

Fig. 2, ZT-30 suelo ordenUltisol.

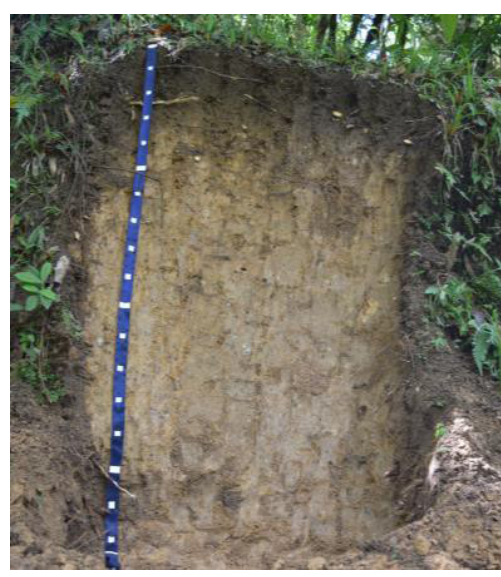

Fig. 3, ZT-133 suelo ordenEntisol.

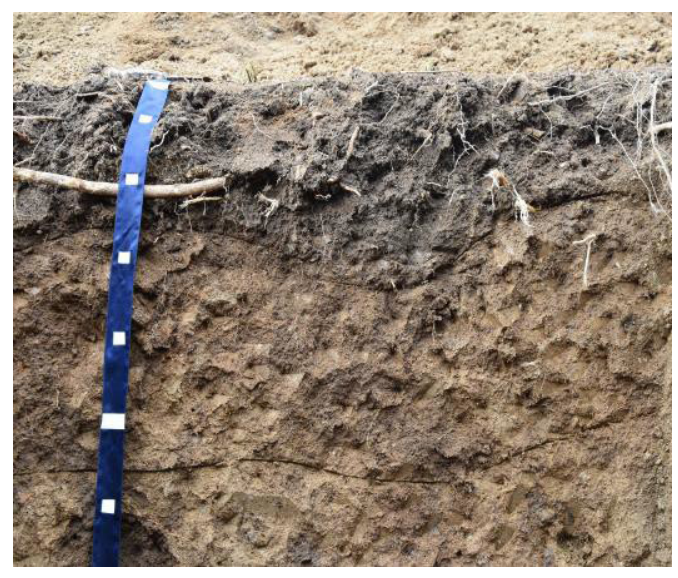

Fig. 4, ZT-104 suelo orden Inceptisol. 


\begin{tabular}{|c|c|}
\hline Parte Baja & $\mathrm{VI} \mathrm{e}_{12} \mathrm{~s}_{2} \mathrm{~d}_{12} \mathrm{C}_{124}$ \\
\hline $\begin{array}{l}\text { Parte Media } \\
\text { Parte Alta }\end{array}$ & $\begin{array}{c}V e_{12} s_{123} d_{2} \\
V e_{12} s_{1234} d_{1} c_{2}, V I e_{12} s_{12} d_{1} c_{14}\end{array}$ \\
\hline
\end{tabular}

\section{CONCLUSIONES}

Con base en el análisis e interpretación de la información obtenida en este estudio de clasificación pedológica, mediante la taxonómica de suelos, se identifica que dentro de la subcuenca del río Zaratí predominan tres órdenes de suelos: Ultisoles, Inceptisoles, y Entisoles, pertenecientes a los grandes grupos Haplustults, Plinthaquults, Epiaquults, Plinthustults, Palehumults, Dystrustepts, Humustepts y Udorthents. En general, los suelos de la SRZ son antiguos, meteorizados, pobres materia orgánica, pobres en bases de intercambio ( $\mathrm{Ca}, \mathrm{Mg}, \mathrm{K})$, arcillosos, y de drenaje lento.

La baja productividad agropecuaria y el alto impacto por las actividades humanas sobre los suelos de la SRZ, así como los fenómenos de degradación, señalan la necesidad de implementar urgentemente prácticas de conservación en el uso de los suelos, para incrementar su calidad agroproductiva y su resiliencia al cambio climático.

Para las tierras onduladas clase $V$ se acuerdo con la capacidad de uso de suelos, ubicadas en la parte media y alta de la SRZ, se recomienda la división de campos y pastoreo rotativo, asimismo, implementación de pastos mejorados, y pasto de corta.

En las tierras clase VI de la parte baja, media y alta de la SRZ, con un relieve fuertemente ondulado y pendientes mayores a $30 \%$, es conveniente siembra de cultivos perennes (frutales, café) y producción forestal que protejan el suelo del impacto directo de la lluvia, promuevan la infiltración del agua y disminuyan el efecto de la escorrentía superficial.

\section{REFERENCIAS}

[1] S. S. Staff, Soil Taxonomy. A basic system of soil classification for making and interpreting soil surveys USDA-SCS, 2 da ed., Washington, D.C., 1999, p. 869.

[2] S. S. Staff, Claves para la Taxonomía de Suelos USDA-NRCS, 12 ed., 2014, p. 643.

[3] FAO-MAG-UNED, Manual de Conservación de suelos y aguas, D. Cubero, Ed., San José: EUNED, 1994, p. 278. 


\section{Autorización y Licencia CC}

Los autores autorizan a APANAC XVIII a publicar el artículo en las actas de la conferencia en Acceso Abierto (Open Access) en diversos formatos digitales (PDF, HTML, EPUB) e integrarlos en diversas plataformas online como repositorios y bases de datos bajo la licencia CC:

Attribution-NonCommercial-ShareAlike 4.0 International (CC BY-NC-SA 4.0) https://creativecommons. org/licenses/by-nc-sa/4.0/.

Ni APANAC XVIII ni los editores son responsables ni del contenido ni de las implicaciones de lo expresado en el artículo. 